Substances Formed by the Interaction of Some Aliphatic Primary Monamines and of Ethylene-Diamine with the Salts of Certain Metals.

\title{
INAUGURAL DISSERTATION
}

FOR THE DEGREE OF DOCTOR OF PHILOSOPHY, ADDRESSED TO THE ACADEMIC FACULTY OF THE UNIVERSITY OF VIRGINIA.

$$
-\mathrm{BY}-
$$

CHARLES JAMES MO0RE.

EASTON, PA.:

Che Chemieal Dublishing Co.

Ig0I.

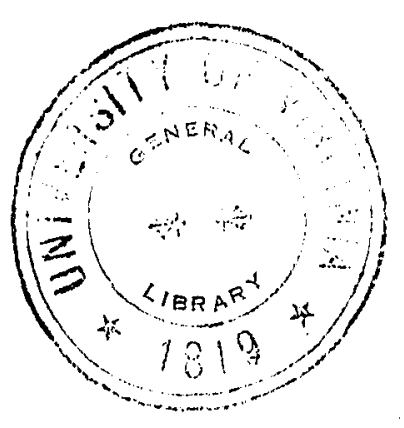




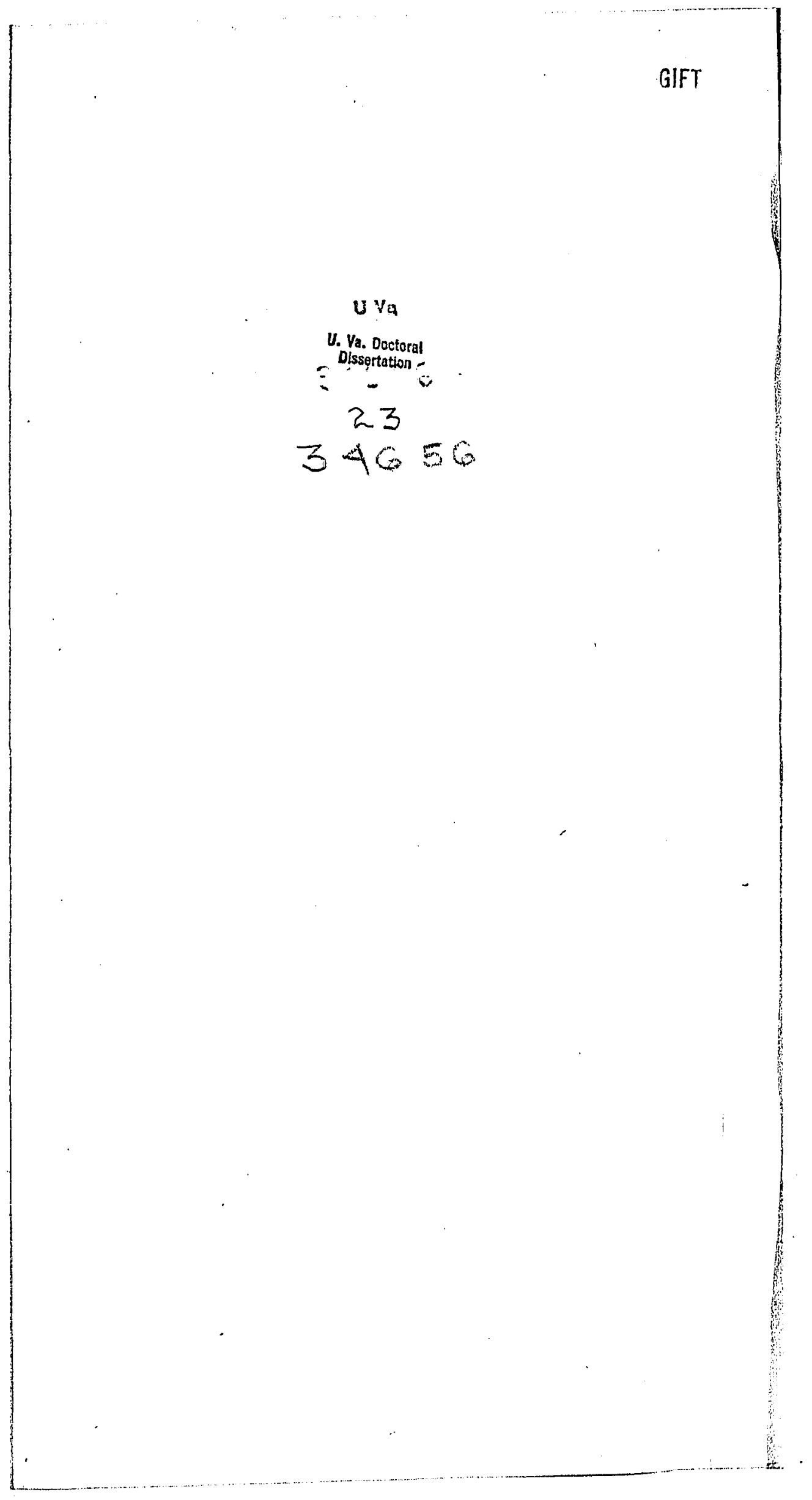




\section{PREFACE.}

The investigation here presented was undertaken at the suggestion of Dr. J. W. Mallet and carried out, in the laboratory of the University of Virginia, under his direction.

I am sincerely grateful to Dr. Mallet for much valuable information, instruction, and inspiration; and to Professor F. P. Dunnington, of the Analytical Department, I am especially indebted for valuable instruction and for the many courtesies he has shown me at all times. The accuracy of the analytical work is due mainly to the excellent methods taught by him.

May I, Igor. 


\section{Introduction,}

Methylamine, preparation of, -

Methods of analysis,

Compounds described, -

Compounds of methylamine with the salts of copper,

Proof of the strong affinity of methylamine for the salts of

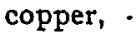

Double halides of methylammonium and copper,

Compound of methylamine with silver nitrate,

Compound of methylamine with mercuric chloride,

Compounds of methylamine with salts of cadmium, Compound of methylamine with nickel sulphate, Compound of methylamine with zinc chloride, Ethylamine, preparation of, Compounds of ethylamine with the salts of copper, Compound of ethylamine with silver nitrate,

Compound of ethylamine with mercuric chloride, Compound of ethylamine with cadmium chloride, Compound of ethylamine with nickel sulphate, Amylamine, preparation of, Compounds of amylamine with cupric chloride, Compound of amylamine with mercuric chloride, Compound of amylamine with cadmium chloride, Compound of amylamine with vickel sulphate, Compounds of amylamine with zinc chloride, Ethylenediamine, preparation of, -

Compound of ethylenediamine with cupric chloride, Compound of ethylenediamine with cadmium chloride, Compound of ethylenediamine zinc chloride,

Double halides of ethylenediammonium and tin, Double halide of ethylenediammonium and cadmium, Double halide of ethylenediammonium and copper, 


\section{SUBSTANCES FORMED BY THE INTERACTION OF SOME ALIPHATIC PRIMARY MONAMINES AND OF ETH- YLENE-DIAMINE WITH THE SALTS OF CERTAIN METALS. \\ INTRODUCTION.}

Although the existence of the anines was definitely predicted by Liebig in $I 842$, they were not actually prepared in the laboratory until early in the year 1849 , when Wurtz discovered that the primary amines could be obtained by decomposing isocyanic ether with caustic potash. Later, in the same year, Hofmann discovered a reaction by which it was possible to prepare not only the primary compounds, but also the secondary and tertiary amines and the quaternary ammonium bases.

While the amines of the lower series are of little commercial value, they are exceedingly interesting from a scientific standpoint, and, indeed, the discovery of them was an event of the greatest importance for the development of organic chemistry. They played a very important part in leading to a "type explanation of the individual classes of compounds." This idea culminated in the theory of Gerhardt which, in turn, found generalization in the extension of the valence theory of Kekule to carbon derivatives. Therefore, we may conclude that had it not been for the discovery of the amines, the advancement in organic chemistry might have been greatly delayed.

The amines are not only interesting in themselves, but also in the variety and number of substances which they form by uniting with other compounds. At this point, it would be well to enumerate some of the more important substances formed by the union of metallic salts with methyl-, ethyl-, and amylamine and ethylenediamine, and also with their hydrochlorides.

A number of double chlorides of methyl- and ethylammo- 
nium and copper, and also mercury have been prepared by Topsoë (Jahresbericht über die Fortschritte der Chemie, I883, 618).

Double halides of ethylammonium and palladium by Reckenschluss (Liebig's Annalen der Chemie, 83, 343).

A compound of ethylamine and mercuric chloride-Köhler (Berichte der deutschen chemischen Gesellschaft, I2, 2323).

Double halides of methylammonium and platinum-Lïdecke (Jahresbericht über die Fortschritte der Chemie, I880, 5II).

Platinoethylamine salts-Jörgensen (Journal für praktische Chemie, 33, 517).

Double halides of cadmium and methylammonium-Ragland; and double halides of tin and methylammonium, and tin and ethylammonium-Cook (American Chemical Journal, December, I899).

Very few compounds have been prepared with amylamine.

Compounds of ethylenediamine and palladium chloride and ethylenediamine and nickel chloride-Kurnakoff (Journal Chemical Society, April, r900).

Compounds of ethylenediamine and cobalt chloride-Jörgensen (Journal für praktische Chemie, 39, 8).

The similarity existing between the compounds of ammonia and of the amines, together with a knowledge of the substances above referred to, led to an extension of the subject and to the preparation of the compounds herein presented.

Preparation of Methylamine.

Methylamine was prepared from acetamide by a process based on Stieglitz's interpretation of the "Beckman rearrangement."

The process, as conducted here, is as follows : 59 grams of acetamide were dissolved in $500 \mathrm{cc}$. of water and to this were added 4I grams of caustic soda, previously dissolved in water, and the mixture was cooled in ice.

Attention should be called to the fact that the above compounds were mixed in a I: I molecular proportion, caustic soda being in slight excess. To the above mixture, bromine was added, drop by drop, from a burette which dipped under 
the surface of the liquid, time being allowed for the solution to cool, as large amounts of heat were produced. Under these conditions, acid bromamide was formed, as is indicated by the following reaction:

$$
\mathrm{CH}_{8} \stackrel{\mathrm{O}}{\mathrm{C}}-\stackrel{\mathrm{H}}{\mathrm{H}}-\mathrm{H}+\mathrm{NaOH}+\mathrm{Br}_{2}=\underset{\mathrm{O}}{\mathrm{O}} \stackrel{\text { CH }}{\stackrel{\mathrm{C}}{1}-\mathrm{NHBr}+\mathrm{NaBr}+\mathrm{H}_{2} \mathrm{O} .}
$$

When all of the acetamide had been thus changed to acid bromamide, the fact was indicated by the formation of sodium hypobromite, from the excess of caustic soda and bromine, which imparted to the colorless liquid a yellow tinge. To this solution was added 120 grams of caustic soda, and onehalf of the mixture was heated, at a time, in a large retort. As only a very moderate temperature is required to start the reaction, and heat appears to be generated in the process, it is best to heat first in a water-bath. Under these conditions the caustic soda is capable of breaking up the acid bromamide, as is indicated in the following reaction:

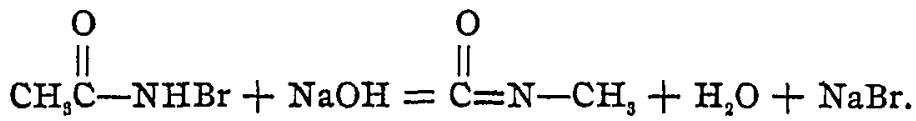

It is here that the "Beckmann rearrangement" takes place, yielding methyl isocyanate which, in turn, is decomposed by more caustic soda into methylamine and sodium carbonate :

$$
\stackrel{\mathrm{O}}{\mathrm{C}}=\mathrm{N}-\mathrm{CH}_{8}+2 \mathrm{NaOH}=\mathrm{NH}_{2} \mathrm{CH}_{3}+\mathrm{Na}_{2} \mathrm{CO}_{9} \text {. }
$$

After the reactions were completed, the contents of the retort were subjected to distillation in order to recover the amine held in solution. The amine is best collected in hydrochloric acid, and the solution of the hydrochloride evaporated to dryness on the water-bath.

The method is rapid and satisfactory, provided we have pure acetamide to start with.

Pure cupric chloride was prepared by dissolving electrolytic 
copper in hydrochloric acid with the addition of the least possible amount of nitric acid, required to effect the solution. The excess of acid was driven off by repeatedly evaporating to dryness and moistening with water.

The water of crystallization was driven off by heating for several hours in an air-bath at $80^{\circ} \mathrm{C}$.

\section{METHODS OF ANAIYSIS.}

Amines were estimated by decomposing the salts with caustic soda and distilling with the aid of steam. The distillate was collected in a known quantity of one-tenth normal sulphuric acid, and the excess of acid was titrated with a corresponding solution of caustic soda.

Copper was determined as the oxide by precipitating it from a boiling solution with sodium carbonate, then adding a hot solution of caustic soda until the green precipitate turned black; the boiling was continued until the amine was expelled. The precipitate was washed three times by decantation, boiling it up each time; it was then transferred to the filter and the washing completed with hot water. This method is quite exact if it is properly carried out.

\section{COMPOUNDS DESCRIBED.}

Her methylamine-cupric chloride.

Tetra methylamine-cupric chloride.

Cupro-methylammonium chloride.

Cupro-methylammonium sulphate.

Tetra methylamine-copper nitrate.

Double halides of methylammonium and copper.

Methylamine-silver nitrate.

Methylamine-mercuric chloride.

Di methylamine-cadmium chloride.

Di methylamine-cadmium sulphate.

Di methylamine-cadmium nitrate.

Tetra methylamine-zinc chloride.

Cupro-ethylammonium chloride.

Tetra ethylamine-copper uitrate.

Ethylamine-silver nitrate.

Ethylamine-mercuric chloride.

Di ethylamine-cadmium chloride.

Di ethylamine-nickel sulphate.

Tetra amylamine-cupric chloride. 
Cupro-amylanimonium chloride. Auylamine-mercuric chloride.

Di amylamine-cadmium chloride.

Di amylamine-nickel sulphate.

Di amylamine-zinc chloride.

Di ethylenediamine-cupric chloride.

Ethylenediamine-cadmium chloride.

Ethylenediamine-zinc chloride.

Etiuylenediamine chlor-stannate.

Ethylenediamine chlor-stannite.

Ethylenediamine chlor-cadmiate.

A double halide of ethylenediammonium and copper.

Attention should be called to the fact, that the above numerical prefixes refer to the number of molecules of amine contained in the substance, and not to the number of alcoholic radicals contained in a single molecule of the amine.

\section{Hex Methylamine-cupric Chloride.}

This compound was prepared by spreading powdered cupric chloride, deprived of its water of crystallization, in a thin layer in a large glass tube, one end of which was connected with a tube dipped under mercury, and the other end, with a small flask containing dry caustic soda. A saturated aqueous solution of methylamine hydrochloride was introduced into the flask, a drop at a time, by means of a tube passing through the stopper and connected with a funnel. By this means, a slow steady stream of dry methylamine was passed over the cupric chloride and under the pressure of about one-fourth of an inch of mercury.

In a few minutes after the current of amine was turned on, the substance nearest the entrance turned from its original brown color to a deep olive-green, and this change of color advanced slowly along the tube, changing successive particles, and when it had reached midway, the portion at the entrance began to turn blue. These colors advanced to the far end of the tube, and the final result was a deep blue, amorphous powder. These changes of color beautifully illustrated the successive formation of different compounds. The stream of amine was kept up sometime after the substance was apparently homogeneous, in order to insure every particle being 
changed. The bulk had increased apparently ten to fifteen times its original volume.

Hex methylamine cupric chloride is quite stable if kept under an atmosphere of dry methylamine, but in dry air it slowly gives off a portion of the amine, and in moist air the decomposition is very much more rapid. It is extremely deliquescent. In a small quantity of water, a portion is decomposed, thereby forming a solution in which the rest is quite soluble, but in larger quantities of water, it is all decomposed.

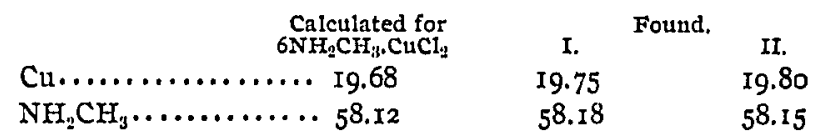

Tetra Methylamine-cupric Chloride.

When hex methylamine-cupric chloride is placed in a sulphuric acid desiccator, it loses one-third of its amine in the course of twenty-four hours, yielding tetra methylamine-cupric chloride, which is very similar in appearance to the compound from which it was derived. It is quite stable so long as it is kept perfectly dry, but it is very deliquescent and decomposes rapidly in moist air. It is also decomposed by the addition of water, a less quantity being required than in the case previously mentioned.

\begin{tabular}{|c|c|c|c|}
\hline & $\begin{array}{l}\text { Calculated for } \\
{ }_{4} \mathrm{NH}_{2} \mathrm{CH}_{3} \cdot \mathrm{CuCl}_{3} \text {. }\end{array}$ & I. & Found. \\
\hline $\mathrm{Cu} . . .$. & .... 24.44 & 24.61 & \\
\hline $\mathrm{NH}_{2} \mathrm{CH}_{3}$ & $\cdots 48.06$ & 48.20 & \\
\hline
\end{tabular}

Cupro-methylammonium Chloride.

If either of the above compounds is exposed to moist air, it will very quickly lose its blue color and as a result of the decomposition, a compound is formed which is apple-green. This substance is insoluble in cold and hot water and in alcohol. It is slowly dissolved by a dilute solution of hydrochloric acid, very probably being first decomposed by it. It may be heated above $100^{\circ} \mathrm{C}$. without decomposition; at a somewhat higher temperature, the amine is slowly driven off, leaving the cupric chloride behind in pure condition. 


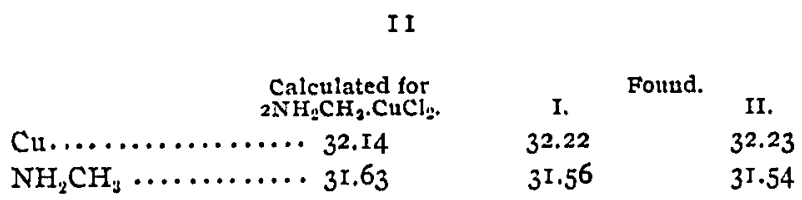

Cupro-methylammonium Chloride.

(Containing one molecule of water.)

When methylamine was passed into a 20 per cent. aqueous solution of cupric chloride, a green precipitate was thrown down, and this was gradually redissolved by an excess of amine, yielding, finally, a clear, deep blue solution. A small amount of heat was developed as the precipitate redissolved. The solution was evaporated to dryness, at the ordinary temperature, in a calcium chloride desiccator.

The result was an amorphous compound, deep blue in color, with many green particles disseminated through the mass. This indicated that decomposition began before the substance was entirely dry. All attempts to obtain a perfectly homogeneous blue compound, by this method, failed. However, analyses were made of the mixture in order to trace, if possible, the stages in the change.

The results of analysis of interior portions of the mass showed a higher per cent. of amine than those of the exterior, and different analyses of the same portion were not sufficiently concordant to establish a formula; but $I$ have many reasons to believe that the blue portion of the mass was of a composition represented by this formula: $6 \mathrm{NH}_{2} \mathrm{CH}_{3} \cdot \mathrm{CuCl}_{2} \cdot \mathrm{H}_{2} \mathrm{O}$. If this does correctly represent the composition of the blue substance, it differs from the compound made by passing dry amine over powdered cupric chloride in that it contains one molecule of water, and that it shows no signs of becoming more stable when one-third of the amine has been lost. The general tendency is to break down into a green compound of this formula: $2 \mathrm{NH}_{2} \mathrm{CH}_{2} \cdot \mathrm{CuCl}_{2} \mathrm{H}_{2} \mathrm{O}$. As will be seen, this differs in composition from a compound previously described, only, in that it contains one molecule of water, and, in fact, the properties of this substance are almost identical with those 0 the compound referred to. 


\begin{tabular}{|c|c|c|c|c|}
\hline & $\begin{array}{l}\text { Calculated for } \\
\text {. }\end{array}$ & & Found. & \\
\hline 11 & .... 29.44 & 29.60 & & 29.5 \\
\hline$H$ & .... 28.97 & 28.90 & & 28.87 \\
\hline${ }_{2} \mathrm{O} \ldots .$. & .... $8.4 \mathrm{I}$ & $8.5^{\circ}$ & & 8.55 \\
\hline
\end{tabular}

The water in the compound referred to above, as in all the following compounds that contain it, was determined by placing a weighed portion of the substance in a glass tube which passed through a water-bath, heated to boiling. One end of this tube was attached directly to a weighed calcium chloride tube and the other end connected with a drying apparatus. A suction pump was attached to the end of the calcium chloride tube, and the tube through which air entered the drying apparatus was drawn out to a capillary bore, in order to diminish the pressure in the tube containing the compound and, at the same time, to allow a steady stream of dry air to pass over the substance. This method was found to be quite accurate.

Cupro-methylammonium Sulphate.

Methylamine was passed into a 20 percent. aqueous solution of cupric sulphate; the green precipitate first thrown down was quite readily redissolved by an excess of the amine, yielding a deep blue solution. Quite a good deal of heat was developed as the precipitate was being redissolved. The solution was evaporated as far as possible in a calcium chloride desiccator, but it was found necessary to complete the drying in a sulphuric acid desiccator.

This compound could not be obtained crystalline; it was of a pale blue color, and showed no tendency to change to green at the ordinary temperature. It was quite deliquescent, and was slowly decomposed by cold water. When heated in a closed tube to $100^{\circ} \mathrm{C}$., amine could be detected, slowly coming off.

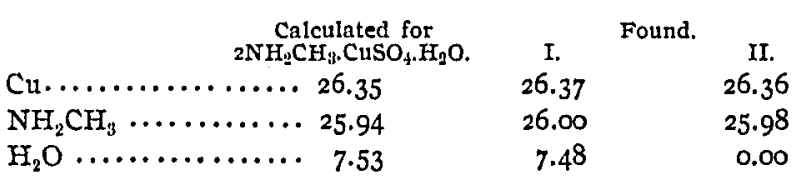

Tetra Methylamine-copper Nitrate.

This compound was obtained by passing an excess of 
methylamine into a 25 per cent. aqueous solution of copper nitrate. No trouble was experienced in completely drying it in a calcium chlorine desiccator.

This compound showed a slight tendency to crystallize in forms, very closely resembling the well-known crystals of sodium platinochloride. It is very stable at the ordinary temperature, showing no tendency to break down into the compound containing only two molecules of the amine. It is moderately deliquescent, deep blue in color, soluble in twentyeight parts of water at the ordinary temperature, and is not decomposed by it. When heated gently, it slowly decomposes; but if the temperature is rapidly raised, it explodes.

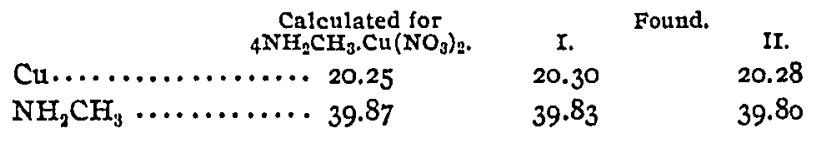

Before leaving the methylamine cupric salts, it would be well to give an account of an experiment by which it was conclusively proved that of the two compounds, ammonia and methylamine, the latter has the stronger affinity for the salts of the metals; and, indeed, this fact proved to be very convenient in the preparation of compounds from solutions of the salts of certain metals and amine, for it was not necessary to separate the methylamine from the small amount of ammonia that was present in it. It was found that if an excess of amine be added to solutions of the salts of certain metals, it will replace all ammonia that might be present. ${ }^{2}$ The presence of the ammonia in methylamine can be readily accounted for by the fact that we often prepare the amine from acetamide that is not absolutely free from ammonium acetate, and under these conditions, the ammonia would be set free and the amine formed simultaneously.

The apparatus used in the preparation of hex methylamine cupric chloride was made use of to pass a mixture of ammonia and methylamine over cupric chloride. The resulting blue compound was analyzed and found to contain ammonia and methylamine in about equal amounts. The compound was then allowed to decompose into a green substance. An

1 This fact has been previously ascertained. 
I4

analysis of this showed that it was cupro-methylammonium chloride and that it contained no trace of ammonia.

Double Halides of Methylammonium and Copper.

In this case it was necessary to have perfectly pure methylamine hydrochloride and this was obtained by taking advantage of the fact that while ammonium chloride is slightly soluble in pure absolute alcohol, it is insoluble in it in the presence of salts of the amines, and methylamine hydrochloride being quite soluble in this solution is easily separated from the ammonium chloride.

Concentrated aqueous solutions of methylamine hydrochloride and cupric chloride were mixed in a $\mathrm{I}: \mathrm{I}$ molecular proportion, the mixture acidified with hydrochloric acid and evaporated in a sulphuric acid desiccator. When the liquid had become quite concentrated, green and brown crystals separated out together, and these could be only partially separated by mechanical neans. It was found, however, that of the two, the brown crystals were much more readily soluble in 90 per cent. alcohol, and by recrystallizing them from it several times, a perfectly homogeneous compound was obtained.

The green crystals were found to be nearly pure cupric chloride, and the brown crystals were a double compound of a $3: 2$ relation. These crystals were tetragonal plates. They were slightly deliquescent, very soluble in cold water and quite soluble in alcohol.

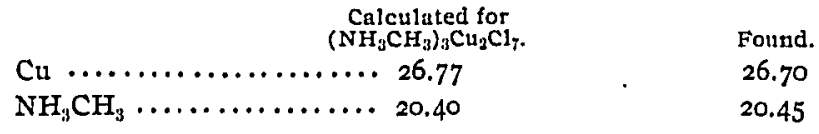

A compound that shows a 2 : I correspondence has already been made and described by Topsoë.

Methylamine-silver Nitrate.

This compound was obtained by passing an excess of methylamine into a ro per cent. aqueous solution of silver nitrate. The precipitate first formed, was very quickly redissolved in excess of amine. The solution was evaporated to dryness in a calcium chloride desiccator. 
This compound crystallized in well defined monoclinic prisms. They were gray in color, slightly deliquescent, soluble in twelve parts of water at the ordinary temperature. When slowly heated they melted at a comparatively low temperature and gradually decomposed. If the temperature be rapidly raised, the compound does not explode as was expected.

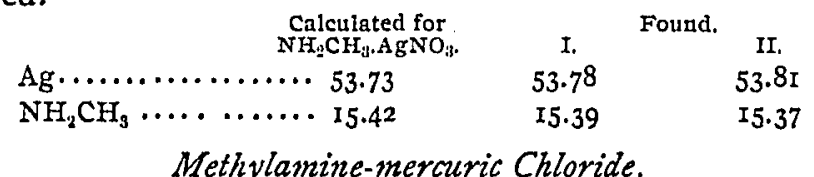

When methylamine was added to a saturated alcoholic solution of mercuric chloride, a heavy, white, granular precipitate was thrown down. This precipitate was insoluble in excess of the amine. The precipitate was allowed to subside and then the supernatant fluid was decanted off. It was washed several times with alcohol, by decantation, and allowed to dry in the air.

This salt was a white, granular powder, insoluble in water, but gradually dissolved in a dilute solution of hydrochloric acid, probably being first decomposed by it. When heated in a closed tube, it slowly decomposed at a low temperature, giving off the amine. It cannot be sublimed.

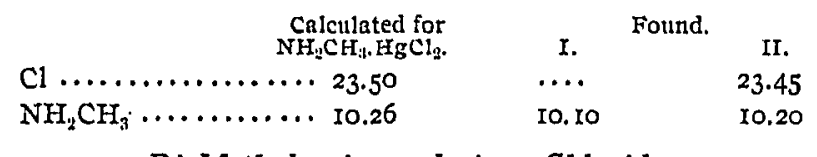

Di Methylamine-cadmium Chloride.

This compound was obtained by passing methylamine into a 20 per cent. aqueous solution of cadmium chloride. The precipitate first formed, was very slowly redissolved by an excess of the amine. A small amount of heat was developed as the precipitate was redissolved. The clear solution was evaporated to dryness in a calcium chloride desiccator.

This compound crystallized in white prismatic forms which were aggregated in masses that presented a star-like appearance. It was slightly deliquescent and was slowly decomposed by water. When heated in a closed tube, it melted, and then quietly gave off the amine. 


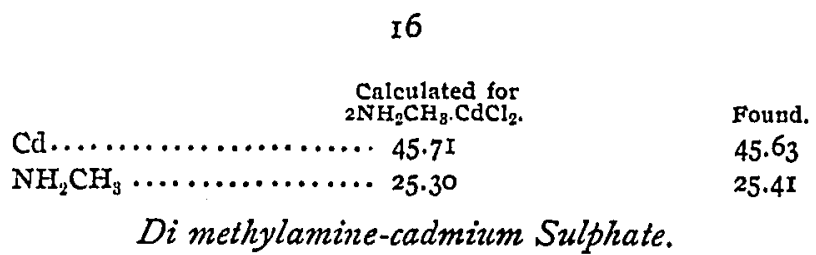

In the preparation of this salt a I5 per cent. solution of cadmium sulphate was used. In other respects the preparation was similar to the chloride.

This substance could not be obtained crystalline. It was quite deliquescent and rapidly decomposed in cold water. When heated in a closed tube, it was perfectly stable at $100^{\circ}$ C. ; at a slightly higher temperature the amine was given off and without first fusing.

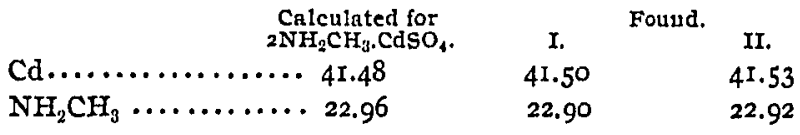

Di methylamine-cadmium Nitrate.

The preparation of this compound was entirely similar to the preparation of the chloride.

It crystallized in beautiful shiny plates. It was slightly deliquescent and was quickly decomposed by water. When heated in a closed tube it melted at a low temperature, began to boil and then exploded violently.

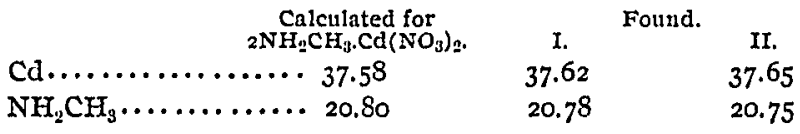

Di methylamine-nickel Sulphate.

This compound was prepared by passing an excess of methylamine into a ro per cent. aqueous solution of nickel sulphate. The precipitate first formed, was very slowly redissolved in excess of the amine, yielding, finally, a clear blue solution. If any heat were given out as this precipitate redissolved, it could not be detected. The solution was evaporated to dryness in a calcium chloride desiccator.

This compound was green in color, amorphous, not deliquescent, insoluble in water and alcohol but was gradually dissolved by dilute acid. When heated, the amine was gradually driven off at a comparatively low temperature. 


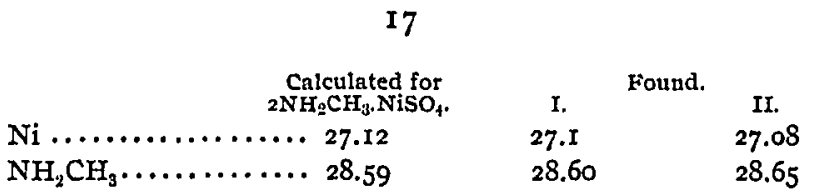

Tetra Methylamine-zinc Chloride.

In the preparation of this salt, a ro per cent. aqueous solution of zinc chloride was used. In all other respects, the process was similar to that used in the preparation of the nickel compound. However, it was found necessary to complete the drying in a sulphuric acid desiccator, and after it had remained even in this for two weeks, it was found to contain so much water that it was thought advisable to leave it several weeks longer, and at the end of this time the results of analysis were perfectly concordant with those first obtained.

This substance was white in color, did not crystallize well from an aqueous solution, and was extremely deliquescent. It was quite soluble in water and moderately soluble in go per cent. alcohol. By evaporating an alcoholic solution of this substance, well defined crystals of the monoclinic system were obtained. These crystals were prismatic in form and their edges and solid angles were much eroded. The prismatic faces were much striated, probably being due to oscillatory combination. They melted at $116^{\circ} \mathrm{C}$.

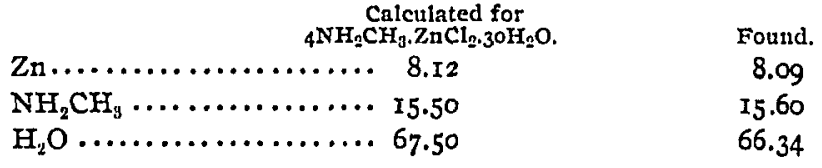

$0.165^{8}$ gram after four hours, heating at $100^{\circ} \mathrm{C}$., weighed 0.1645 .

$0.165^{8} \mathrm{gram}$ after two hours, heating at $\operatorname{Ir} 6^{\circ} \mathrm{C}$., weighed 0.1615 .

$0.165^{8}$ gram after two hours, heating at $140^{\circ} \mathrm{C}$., weighed $0.055^{8}$

After being thus beated the compound analyzed as follows :

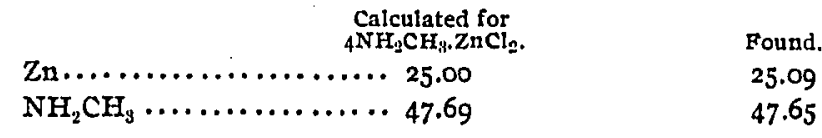




\section{I8}

Compounds with Ethylamine.

Ethylamine was prepared by the original Würtz process, which consists in distilling a dry mixture of potassium isocyanate and ethyl potassium sulphate, and decomposing the cyanic ester thus formed by heating with a strong solution of caustic potash.

\section{Cuproethylammonizum Chloride.}

This compound was obtained by passing an excess of ethylamine into a 20 per cent. aqueous solution of cupric chloride, and evaporating the clear blue solution in a calcium chloride desiccator. The blue compound which first separated out could not be obtained homogeneous, so it was allowed to decompose into the more stable green form.

This compound was in all respects similar to the corresponding compound with methylamine. It was amorphous, apple-green in color, slightly deliquescent, and insoluble in water. In dilute acids, it dissolved quite readily. When heated in a closed tube, the amine began to be driven off very slowly at $100^{\circ} \mathrm{C}$. and at $130^{\circ}$ it came off quite rapidly.

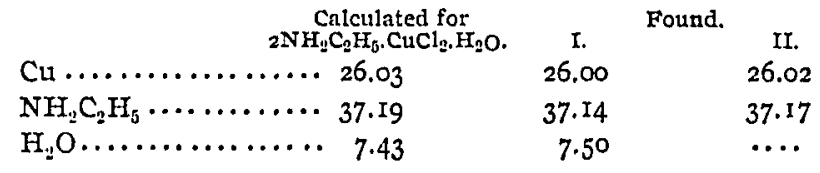

Tetra Ethylamine-copper Nitrate.

This compound was obtained by the same process used in the preparation of the chloride, except in that a 25 per cent. aqueous solution of copper nitrate was used.

This substance crystallized fairly well in prismatic forms with pointed ends. It was of a deep blue color and was quite stable at the ordinary temperature. When heated gently, it melted and slowly gave off the amine. When the temperature was rapidly raised, it exploded. It was moderately deliquescent and quite soluble in cold water.

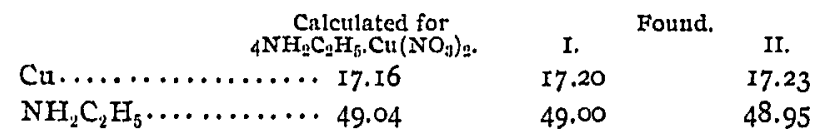


I9

Ethylamine-silver Nitrate.

This salt was prepared by passing ethylamine into a ro per cent. aqueous solution of silver nitrate until the precipitate first formed, was entirely redissolved, and then evaporating the solution to dryness in a calciun chloride desiccator.

This compound crystallized fairly well in monoclinic prisms which were gray, slightly deliquescent, and quite soluble in cold water. It behaved in all respects similar to the corresponding compound with methylamine.

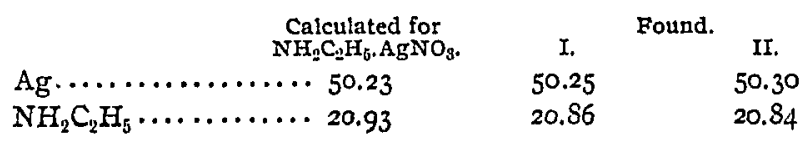

Ethylamine-mercuric Chloride. ${ }^{1}$

The process used in the preparation of this substance was in every way similar to that used in the preparation of the corresponding compound with methylamine and, indeed, these two substances were so nearly alike in appearance and properties that a careful quantitative analysis would be required in order to distinguish them.

When heated in a closed tube this salt began to decompose at $160^{\circ} \mathrm{C}$.

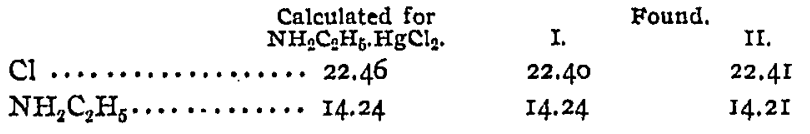

Di Ethylamine-cadmium Chloride.

This was prepared by passing ethylamine into a I 5 per cent. aqueous solution of cadmium chloride until the precipitate redissolved, and evaporating the solution to dryness as described previously.

The salt could not be obtained crystalline, but in other respects it resembled very closely the corresponding compound with methylamine. It was stable at $175^{\circ} \mathrm{C}$. At a somewhat higher temperature it melted, and the amine was driven off.

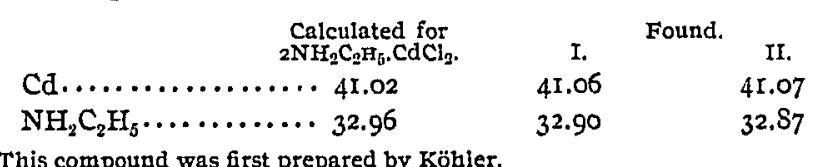

1 This compound was first prepared by köhler.

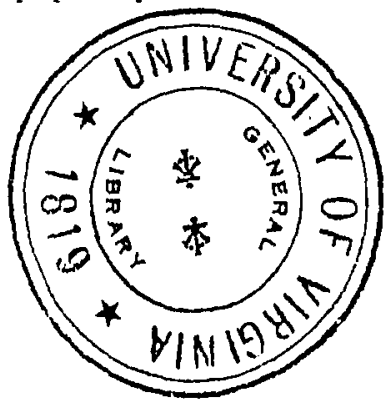


20

Di Ethylamine-nickel Sulphate.

This compound was prepared by the same process used to prepare di methylamine-nickel sulphate, and the two were similar in every respect.

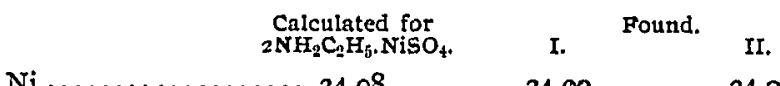

$$
\begin{aligned}
& \mathrm{Ni} \ldots \ldots \ldots \ldots \ldots \ldots, 24.08 \quad 24.00,24.03 \\
& \mathrm{NH}_{2} \mathrm{C}_{2} \mathrm{H}_{5} \ldots \ldots \ldots \ldots . \cdots 36.73 \quad 36.77 \quad 36.76
\end{aligned}
$$

Amylamine was prepared by the Wïrtz process.

Tetra Amylamine-cupric Chloride.

Pure amylamine was added to a 15 per cent. aqueous solution of cupric chloride, and in this case, the green precipitate first formed, did not redissolve as readily in excess of this amine as it did in either methyl-or ethylamine. Finally, however, a clear, deep blue solution was obtained, and this was poured over a widely extended surface and evaporated rapidly in a calcium chloride desiccator.

The substance thus prepared proved to be tetra amylaminecupric chloride. It was of a rather pale blue color, not very deliquescent, and quite soluble in water. It very slowly decomposed into cuproamylammonium chloride at the ordinary temperature, but at $50^{\circ} \mathrm{C}$. the decomposition was quite rapid.

$$
\begin{aligned}
& \begin{array}{l}
\text { Calculated for } \\
4 \mathrm{NH}_{2} \mathrm{C}_{6} \mathrm{H}_{11}, \mathrm{CuCl}_{2}, \mathrm{H}_{2} \mathrm{O}
\end{array} \\
& \mathrm{Cu} \ldots \ldots \ldots \ldots \ldots \ldots \ldots \ldots \times 12.60 \quad{ }^{4 \mathrm{NH}_{2} \mathrm{C}_{6} \mathrm{H}_{11}, \mathrm{CuCl}_{2}, \mathrm{H}_{2} \mathrm{O} .} \quad \mathrm{I} 2.65 \\
& \mathrm{NH}_{2} \mathrm{C}_{5} \mathrm{H}_{11} \ldots \ldots \ldots \ldots \ldots .69 .60 \quad 69.48 \\
& \mathrm{H}_{2} \mathrm{O} \ldots \ldots \ldots \ldots \ldots \ldots \ldots, 3.60 \ldots
\end{aligned}
$$

Cuproamylammonium Chloride.

This was obtained by heating tetra amylamine-cupric chloride in an air-bath for two hours at $50^{\circ} \mathrm{C}$.

It was amorphous, green, insoluble in water, but readily dissolved in dilute mineral acids. When heated in a closed tube, it melted at $109^{\circ}$, then became black, and amine was driven off. 
2 I

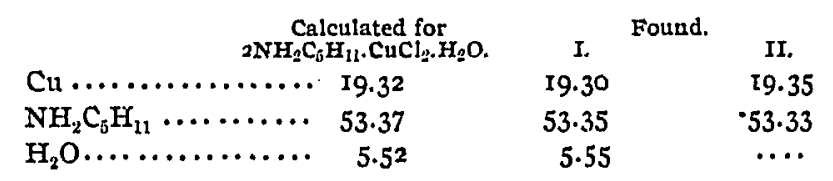

A silver salt with amylamine was prepared, but it was so prone to decompose that an analysis of it could not be made before decomposition began.

\section{Amylamine-mercuric Chloride.}

This compound was obtained by adding a slight excess of amylamine to a saturated alcoholic solution of mercuric chloride. For this purpose 92 per cent. alcohol was used. The heavy white precipitate was allowed to subside and the supernatant liquid was then decanted off. The rest of the alcohol was allowed to evaporate and the precipitate was dried in a desiccator.

This compound could not be obtained crystalline. It was a cheese-like substance and held on to surplus amine so tenaciously that it was found necessary to heat it for four hours in an air-bath at $75^{\circ} \mathrm{C}$. in order to get rid of this excess of amine. The results of analyses were then quite concordant. This substance was quite readily soluble in hot dilute hydrochloric acid. When heated in a closed tube, it began to decompose at $150^{\circ}$ without melting.

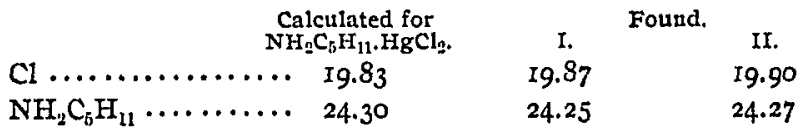

Di Amylamine-cadmizm Chloride.

This compound was prepared by the same process used in the preparation of the corresponding compounds with ethyland methylamine.

This substance was white, amorphous, and very slightly deliquescent. It was necessary to heat it to $75^{\circ}$ in order to get rid of excess of amine. It was insoluble in cold and hot water, but was easily dissolved by dilute hydrochloric acid. When heated in a closed tube, it was perfectly stable at $175^{\circ}$ C. At a higher temperature it melted, and the amine was driven off. 


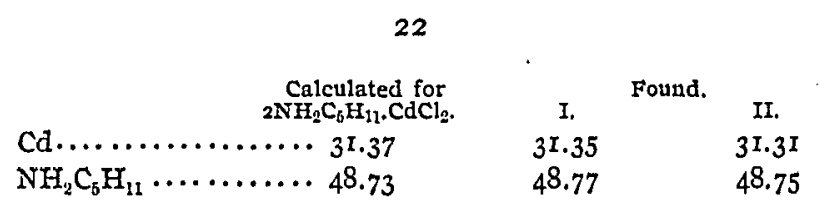

Di Amylamine-nickel Sulphate.

When amylamine was added to a 15 per cent. aqueous solution of rickel sulphate, a green precipitate was thrown down, and this was very slowly redissolved by an excess of amine, yielding, finally, a clear violet solution, and this was evaporated to dryness in the usual manner.

The resulting compound was amorphous, green in color, insoluble in water and alcohol, but dissolved in hot dilute hydrochloric acid. When heated in a tube, it began to decompose at $105^{\circ}$ without melting.

\begin{tabular}{|c|c|c|c|}
\hline Calculated for & & Found. & \\
\hline Ni $\ldots \ldots \ldots \ldots \ldots \ldots \ldots, 17.93$ & 17.90 & & 17.91 \\
\hline $\mathrm{NH}_{2} \mathrm{C}_{5} \mathrm{H}_{11} \ldots \ldots \ldots \ldots, 52.88$ & 52.94 & & $52.9 \mathrm{I}$ \\
\hline
\end{tabular}

This compound was prepared by adding amylamine to a io per cent. solution of zinc chloride, until the precipitate first formed, redissolved, and then evaporating the solution to dryness.

It showed a slight tendency to crystallize in needles, which were snow-white, insoluble in water, but readily dissolved in dilute acid. When heated in a tube decomposition began at $85^{\circ}$.

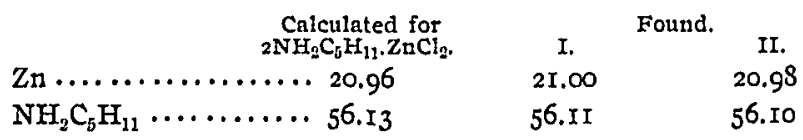

COMPOUNDS WITH ETHYLENEDIAMINE.

Preparation of Ethylenediamine.

About two-thirds of the theoretical yield of the hydrochloride was obtained by heating 42 grams of ethylene chloride with 33 per cent. aqueous ammonia ( 5 ro cc.) in sealed tubes at $I I 7^{\circ}$ for five hours. The contents of the tubes were then evaporated until crystals began to form, and then three vol- 
umes of absolute alcohol were added in order to precipitate the ethylenediamine hydrochloride. The crystals were washed with absolute alcohol until the washings ceased to color Nessler's solution.

The hydrate of the free base was obtained by the addition of ireshly fused and powdered caustic soda to the hydrochloride, then adding soda-lime and distilling. The anhydrous base may be obtained from this hydrate by again heating with fused soda in a sealed tube for several hours.

A much better yield of ethylenediamine hydrochloricle was obtained by heating ethylene chloride with alcoholic ammonia in sealed tubes at $100^{\circ}$ for two hours.

\section{Di Ethylenediamine-cupric Chloride.}

This compound was prepared by adding ethylenediamine hydrate to a 20 per cent. aqueous solution of cupric chloride until the precipitate first formed, was redissolved. The solution was evaporated to dryness in a sulphuric acid desiccator.

This substance was a deep ultramarine violet in color, and showing no tendency whatever to decompose into a green compound similar to those obtained with the monamines. It was very stable, quite deliquescent, and could not be obtained crystalline. It was quite soluble in cold water. When heated in a closed tube, if the temperature be rapidly raised, it shows slight signs of melting at about $105^{\circ}$, but if the water be first driven off, it melts at a much higher temperature and then slowly decomposes.

$$
\begin{aligned}
& \begin{array}{l}
\text { Calculated for } \\
2 \mathrm{C}_{2} \mathrm{H}_{4}\left(\mathrm{NH}_{2}\right)_{2} \cdot \mathrm{CuCl}_{2} \cdot 2 \mathrm{H}_{2} \mathrm{O} . \\
\text { I. Found. II. }
\end{array} \\
& \text { Cu.............. 21.72 2I.77 } \\
& \mathrm{C}_{2} \mathrm{H}_{4}\left(\mathrm{NH}_{2}\right)_{2} \ldots \ldots \ldots \ldots 4 \mathrm{I}_{3} 3^{8} \quad 4 \mathrm{I} .3 \mathrm{I} \\
& \mathrm{H}_{2} \mathrm{O} \ldots \ldots \ldots \ldots \ldots, \mathrm{I} 2.4 \mathrm{I} \quad \mathrm{I} 2.5 \mathrm{O} \quad \ldots
\end{aligned}
$$

Ethylenediamine-cadmium Chlorde.

The process used in the preparation of this compound was similar to that used to prepare the corresponding salt with copper. A ro per cent. aqueous solution of cadmium chloride was used.

This substance crystallized in needles. It was very stable, readily soluble in water, white, not deliquescent. When 
heated in a closed tube, it melted at a moderately low temperature and then decomposed.

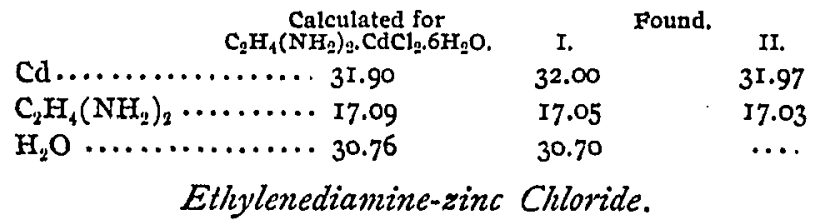

This was obtained by the same process used above. A I5 per cent. aqueous solution of zinc chloride was used.

This compound crystallized in transparent monoclinic prisms which had a glassy luster. It was readily soluble in water. When heated in a closed tube, it melted at a moderately low temperature and decomposed. At the ordinary temperature it was very stable.

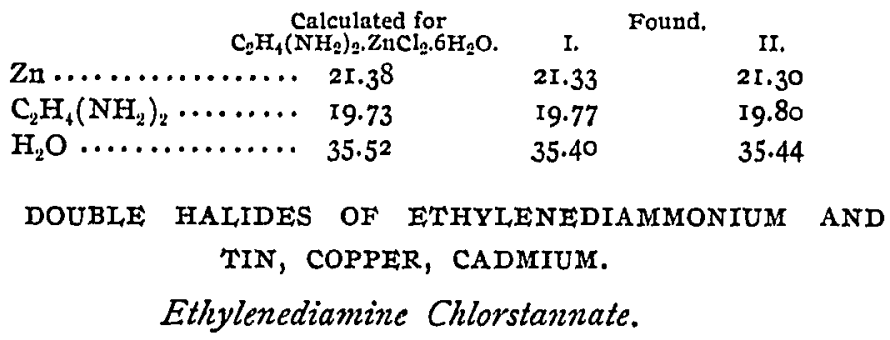

2:I. This salt was obtained by mixing concentrated aqueous solutions of ethylenediamine hydrochloride and stannic chloride in a 2: I molecular proportion, acidifying with hydrochloric acid, and evaporating in a sulphuric acid and caustic soda desiccator. When the liquid had become quite concentrated, large, transparent, hexagonal tables separated out. Some of these measured $3.6 \mathrm{~mm}$. across the base. The mother-liquor was drained off and the crystals were washed with water, pressed between the folds of smooth drying paper and then air-dried.

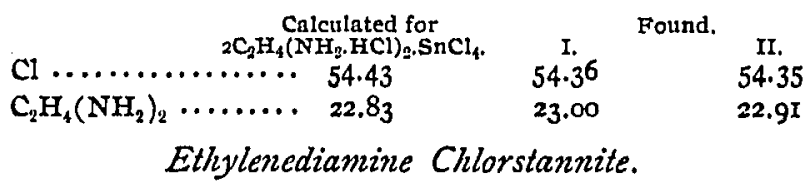

I:I Ethylenediamine hydrochloride and stannous chloride 
in concentrated aqueous solutions were mixed in a $I: I$ proportion and the solution evaporated as in the previous case. The liquid was not as concentrated when the crystals began to separate out as it was in the case with the stannic salt. The crystals were large, transparent, triclinic plates. Some of these plates measured about $4 \mathrm{~mm}$. across the face. They were separated from the mother-liquor, washed with water, and dried in the air.

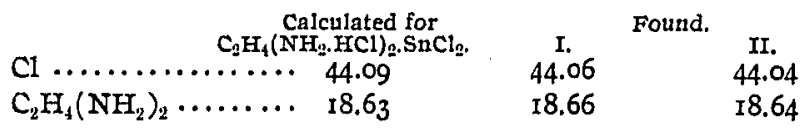

Ethylenediamine Chlorcadmiate.

I:I Concentrated solutions of the two salts were mixed in a I:I and a 2:I proportion, and in this case it was found advantageous to have present quite a good deal of free hydrochloric acid to prevent some form of cadmium from separating out and rendering the crystals translucent. The crystals obtained from each solution were found to be identical. They were transparent, well-formed prisms of the triclinic system. They were exceedingly soluble in water, but very much less soluble in alcohol, so they were washed free of the motherliquor with alcohol.

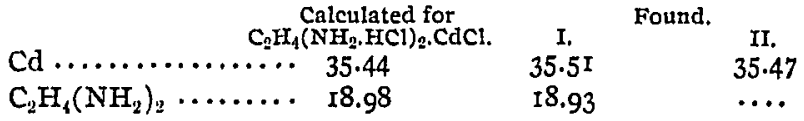

A I : I compound with copper was obtained by mixing concentrated solutions of the two salts in a 2 : I proportion, and evaporating as above described. The crystals did not begin to separate out until the liquid was very concentrated. The mother-liquor was drained off, and it was found necessary to recrystallize them twice before they could be obtained perfectly pure. They were blue monoclinic prisms, capped by pyramids. The excess of ethylenediamine hydrochloride

\begin{tabular}{|c|c|c|}
\hline 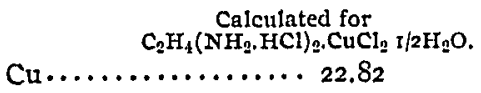 & $\begin{array}{l}\text { I. Found. } \\
22.79\end{array}$ & $\begin{array}{c}\text { II. } \\
22.80\end{array}$ \\
\hline $\mathrm{C}_{2} \mathrm{H}_{4}\left(\mathrm{NH}_{2}\right)_{2} \ldots \ldots \ldots \ldots 2 \mathrm{I} .73$ & 21.75 & .... \\
\hline $\mathrm{H}_{2} \mathrm{O} \ldots \ldots \ldots \ldots \ldots \ldots \ldots, 3.26$ & 3.30 & $\cdots$ \\
\hline
\end{tabular}
separated out almost in pure form. 


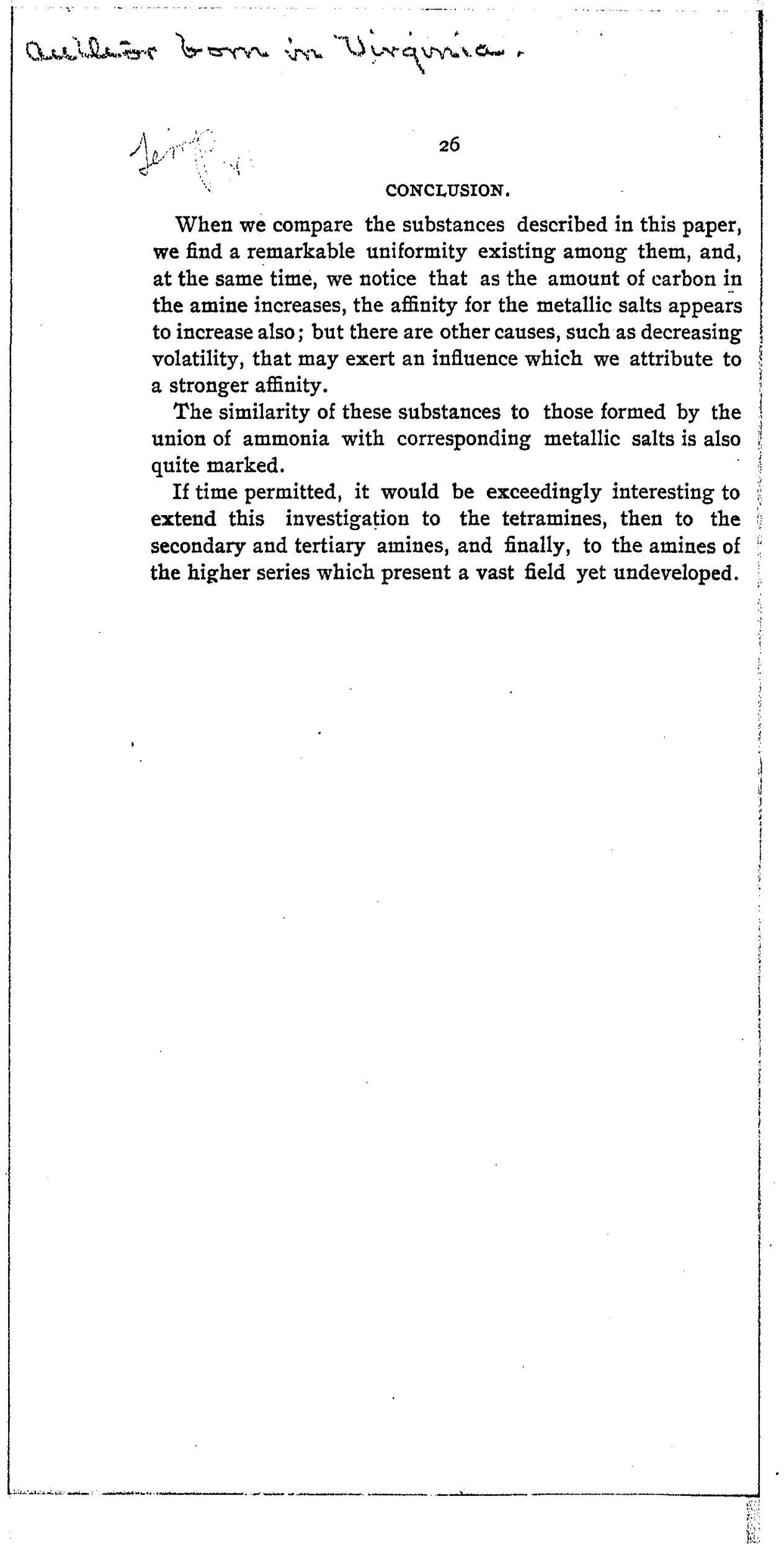

